\title{
Negative Stress Margins - Are They Real?
}

\author{
Ivatury S. Raju ${ }^{1}$ \\ NASA Langley Research Center, Hampton, Virginia \\ Darlene S. Lee ${ }^{2}$ \\ Jet Propulsion Laboratory, Pasadena, California \\ Michael Mohaghegh ${ }^{3}$ \\ The Boeing Company, Seattle, Washington
}

\begin{abstract}
Advances in modeling and simulation, new finite element software, modeling engines and powerful computers are providing opportunities to interrogate designs in a very different manner and in a more detailed approach than ever before. Margins of safety are also often evaluated using local stresses for various design concepts and design parameters quickly once analysis models are defined and developed. This paper suggests that not all the negative margins of safety evaluated are real. The structural areas where negative margins are frequently encountered are often near stress concentrations, point loads and load discontinuities, near locations of stress singularities, in areas having large gradients but with insufficient mesh density, in areas with modeling issues and modeling errors, and in areas with connections and interfaces, in two-dimensional (2D) and three-dimensional (3D) transitions, bolts and bolt modeling, and boundary conditions. Now, more than ever, structural analysts need to examine and interrogate their analysis results and perform basic sanity checks to determine if these negative margins are real.
\end{abstract}

\section{INTRODUCTION}

The current trend in the design process is an increasing reliance on structural modeling and simulation to assess local stress states and evaluate margins of safety. There is also a tendency to perform three-dimensional (3D) analyses under the pretense that detailed 3D models inherently provide higher fidelity and more accuracy than two-dimensional (2D) and shell models. In addition, aerospace structural components inherently have local stress concentrations, free edges, skin-stiffeners, varying thickness shells, etc. Global- or system-level structural models for loads and deflections often include connections

\footnotetext{
${ }^{1}$ NASA Technical Fellow for Structures, NASA Engineering and Safety Center. Fellow AIAA, Member ASME, Member ASCE.

${ }^{2}$ Group Supervisor.

${ }^{3}$ Boeing Technical Fellow, Associate Fellow AIAA, Member ASCE.
} 
between and among finite elements of different dimensionality (e.g., beam element connected to a plate/shell/solid element) that act as point loads.

Finite element stress analysis results from these situations are frequently cited and used to evaluate margins of safety. Quite often negative stress margins are encountered in these analyses. The negative margins raise questions about the adequacy of the structural design and may in fact initiate separate independent assessments of the design, a redesign of the component(s), or both. Alternatively, these stress values may be prescribed as input for a life-prediction analysis using tools such as NASGRO ${ }^{1}$ and $\mathrm{AFGRO}^{2}$, and the predicted outcome may be inadequate design life driven in part by artificially high local stress values. As a consequence, schedule delays may result and costs may increase due to perceived necessity to redesign.

In some but not all cases, these negative stress margins computed using local stresses are inaccurate and are artifacts of modeling and analysis. The purpose of this paper is to discuss various situations wherein negative stress margins are not real, to raise the awareness about how to use finite element stress output, and to discuss margin of safety calculations. The intent of this paper is to increase the analysts' awareness of such occurrences, as reliance on analysis becomes more and more common, and to encourage seasoned analysts to invest time in mentoring their junior colleagues. Also, the intent of this paper is to inform and raise a flag based on some lessons learned. For early-career stress analysts, the paper serves as motivation for learning and/or relearning the theory of elasticity and other continuum mechanics approaches as well as encouraging the use of classical analyses techniques that have been validated by over 40 years in aerospace applications. These techniques can be found in $\mathrm{Bruhn}^{3}$ and Astronautics Structures Manuals ${ }^{4-6}$.

In aerospace structures, margin assessments are performed usually for strength, stiffness, stability (global buckling, local buckling, crippling), fatigue and fracture. In this paper, the discussion of the margin assessments is limited to strength and stiffness using numerical analyses such as the finite element method. Most of the discussion in this paper is generally applicable to both isotropic (metallic) materials and anisotropic (composite) materials. Also, an attempt is made to discuss special issues related to composites.

The paper is organized as follows. First, factor of safety (FOS) and margin of safety (MOS) are defined and discussed along with definitions of ultimate, yield, and limit loads. Variables and details involved in the margin of safety calculations are discussed. Second, the general analysis process and the building-block approaches in the analysis are presented. Next, the areas where negative margins are frequently encountered in numerical analyses such as finite element (FE) analysis are discussed. The paper concludes with a general discussion along with advice to early-career analysts. 


\section{FACTORS OF SAFETY (FOS) AND MARGIN OF SAFETY (MOS)}

To account for uncertainties and unknowns, including material variations, part and assembly variations, analysis uncertainties, etc., a structural member must be designed to carry a load considerably larger than the maximum expected applied load. This design load is established when the maximum expected applied load is multiplied by a FOS. In the 1930's there was ambiguity among the definitions used for design load, expected load and applied loads. Therefore, the U.S. Army Air Corps established the following definitions summarized in Table 1 that are used today in the aerospace industry.

Table 1. Terminology Definitions

\begin{tabular}{|l|l|}
\hline \multicolumn{1}{|c|}{ Term } & \multicolumn{1}{c|}{ Definition } \\
\hline Limit Load & $\begin{array}{l}\text { Maximum expected load on the structure in its life } \\
\text { time and in the entire fleet. }\end{array}$ \\
\hline Ultimate Load" & $\begin{array}{l}\text { Product of the Limit Load times the Ultimate } \\
\text { Factor of Safety (FOS } \\
\text { which a structure is designed for ultimate strength } \\
\text { and must be less than the Allowable Ultimate } \\
\text { Load. }\end{array}$ \\
\hline Yield Load & $\begin{array}{l}\text { Product of the Limit Load times the Yield Factor } \\
\text { of Safety (FOS } \\
\text { structure is designed for yield strength and must be } \\
\text { less than the Allowable Yield Load. }\end{array}$ \\
\hline $\begin{array}{l}\text { Allowable Ultimate Load } \\
\text { The highest load that will not cause material } \\
\text { failure. }\end{array}$ \\
\hline $\begin{array}{l}\text { Allowable Yield Load } \\
\text { are really the ultimate capability of the structure and the yield capability of the } \\
\text { structure that does not result in detrimental deformation, respectively.) }\end{array}$ \\
\hline $\begin{array}{l}\text { 1'Ultimate Load is also often referred to as "Ultimate Design Load" or "Design } \\
\text { plastic deformation. }\end{array}$ \\
\hline
\end{tabular}

The Factors of Safety for ultimate and yield loads are defined as:

FOS $_{\text {ult }}=\frac{\text { Ultimate Load }}{\text { Limit Load }}$ 
FOS $_{\text {yield }}=\frac{\text { Yield Load }}{\text { Limit Load }}$

Note that equation (1) shows the Factor of Safety as a derived value. In typical aerospace applications, the Factors of Safety are requirements based on whether the structure being analyzed is a structure to be test verified or is strictly verified by analyses as well as other risk factors including the brittleness of the structural material, the type of loading, the consequence of failure, etc.

The aerospace industry also uses an additional term called the Margin of Safety (MOS). The MOS relates the design load to the allowable load.

$$
\begin{aligned}
\operatorname{MOS}_{\mathrm{ult}}= & \frac{\text { Allowable ultimate load }}{\text { Ultimate load }}-1 \\
= & \frac{\text { Allowable ultimate load }}{\text { Limit load*FOS }}-1
\end{aligned}
$$

and

$$
\begin{aligned}
\operatorname{MOS}_{\text {yield }} & =\frac{\text { Allowable yield load }}{\text { Yield load }}-1 \\
& =\frac{\text { Allowable yield load }}{\text { Limit load*FOS } \text { yield }_{\text {I }}}-1
\end{aligned}
$$

When the $\mathrm{MOS}_{\text {ult }}$ equals zero, the Allowable Ultimate Load, or capability, equals Ultimate Load, the load for which the structure was designed.

Figure 1 schematically presents the loads defined above and the relationship between $\mathrm{FOS}_{\text {ult }}$ and MOS $\mathrm{Mlt}_{\text {. }}$ The load is plotted against the stress of a linear elastic structure.

Margin of safety is thus a measure of the remaining load-carrying capacity of a structure existing under an applied load condition. A margin of safety typically serves two functions ${ }^{7}$. First, the algebraic sign of the margin of safety indicates whether or not the structure is safe with respect to the applied loads. Second, the magnitude of the margin of safety indicates the amount the applied loads can be increased without exceeding the stipulated yield or ultimate capability of the structure. Therefore, the analyst must exercise judgment in maintaining some minimum $\mathrm{MOS}_{\text {ult }}$ to account for part variations, assembly variabilities, and the uncertainties in and limitations of the analyses. 
Since the relationship between load and stress is often linear, many engineering texts will define the FOS and MOS with respect to stress as opposed to loads. On the other hand, if a nonlinear relationship between the applied loads and the resulting stresses exists, a margin of safety calculated with respect to the loads, in general, will not be the same as a margin calculated with respect to the stresses. Typical cases are redistribution of loads due to changes in stiffness of the redundant structure, operation in the post-buckled range, and other nonlinear behaviors.

Margin of safety (MOS) on stresses are reported in the analyses using the equation that is similar to the load equation (Eq. 2 and 3 ) as

MOS $=\frac{\text { Allowable stress }}{\text { FOS } \times \text { Calculated stress }}-1$

in which the allowable stress is usually developed from uni-axial material testing. The calculated stress is the stress computed using numerical methods such as the finite element (FE) method subjected to the loading condition under consideration. Various loading conditions such as mechanical, thermal, pressure, etc. loads are considered in the FE analyses. The calculated stress could be a maximum principal stress, maximum shear stress, a von Mises stress, etc. depending on the failure scenario that is postulated by the analyst. When dealing with multiaxial stress states, invariant stress measures such as von Mises stresses are usually preferred. While evaluating various failure scenarios, analysts need to evaluate the MOS for each of these scenarios and determine the most constraining scenario and then report the MOS for that scenario.

\section{Design Allowables}

Computed stresses or strains must be compatible with the allowable stress, the Design Allowable (DA) value that is used in the MOS calculation. For static strength analysis, the primary requirement is a consistent process for establishing rational, compatible, and validated values for both the numerator and the denominator in Eq. (4). Such a process requires a thorough understanding of all design requirements, the physical response of the structure under the applied loading, the anticipated nature of the failure modes being addressed in each analysis step, and a basic understanding of the material properties and allowables used to perform the analyses. In addition, understanding the sensitivities of the predicted stress states to variations in these input parameters is needed.

Too often in formulating a new analysis approach, emphasis is placed on generating a stress or strain (possibly with the use of a detailed FE analysis) response to the applied load, with far too little thought given to the mode of failure that is being evaluated and its associated and appropriate Design Allowable. The key characteristic of a computed structural response used in a MOS calculation is that it must be compatible with the DA value.

Design allowables are all based upon test-derived material properties that have a 
statistical basis (i.e., A-basis or B-basis allowable). In some cases, a DA will simply be a material allowable for a specific material, environment (i.e., temperature, humidity), heat treatment, stock form (e.g., bar, sheet, plate), and grain orientation (i.e, Ftu LT). In this case, the design values are sometimes referred to as Material Design Allowables (MDA) since the material values are largely independent of the configuration of the structure that they are being applied to and are based upon material specification requirements/attributes for the product form (i.e., plate, bar, extrusion, temper or condition, and size). Also, part orientation relative to the stock material may be important (grain orientation). In most cases, modifications to material allowables are required. These modifications may be fairly simple (factors to account for elevated temperatures, or to allow for repair attachments), but many involve complex calculations that are a substantial part of the analysis method and may involve FE analysis (stiffened panel general stability, allowables for various post buckled failure modes, etc.). All allowable modifications require test validation. In many cases, sufficient element or coupon tests are conducted to generate modification factors that are compatible with statistical A- or B-basis allowable (i.e, thermal corrections, open-hole compression, filled hole tension corrections for composites, etc.). This is true for fastener and joint allowables. For some of the more complex allowable calculations, generally referred to as design values, such as stiffened panel general stability, or fuselage skin and stringer shear and compression interaction, sufficient large-scale testing is required to show that the analysis method is accurate or conservative for the design space in which it is used. This means that for a new design or new materials, additional testing may be required to validate the design value calculation. At the other extreme compared to MDA is an allowable that is only valid for a specific structural configuration which is called a Point Design Allowable. These allowables are valid only for the structure tested (which may involve a mix of materials). While the number of tests required may be based on the design conditions, structural complexity, and the actual property being tested, a sufficient number of tests are still required to establish a statistical basis.

The statistical basis that must be used for derivation of the allowable may depend on the type of analysis, the structural configuration, and the type of loading. For ultimate analysis of structures with redundant load paths, B-basis allowables are used. For ultimate analysis of single load path structures, A-basis allowables are required. For residual strength analysis, use of typical (average) allowable is generally permissible. Adjustments to the basic DA are often required to account for environmental influences (temperature, moisture), undetectable damage (Barely Visible Impact Damage, BVID), undetectable manufacturing defects (such as flaws or fiber wrinkles exhibited by a stable and repeatable manufacturing process), or residual stresses due to fabrication or assembly (i.e., residual thermal stresses due to welding or curing, fit-up stresses due to assembly). 


\section{ANALYSIS PROCESS}

Analysis of a complex aerospace structure or a structural component consists of several well defined steps. This process is described in this section. After this description, a building-block approach that is recommended by experienced analysts is presented and discussed.

The general steps followed in an analysis process are:

1. Define the Purpose of Analysis

2. Understand the Problem

3. Understand the Design Requirements

4. Research Existing Approaches

5. Formulate the Analysis Plan

6. Select the Stress Analysis Method

7. Execute the Analysis

8. Evaluate Analysis Results

9. Document Analysis and Results

\section{Building-Block Approach and Analysis Models}

While the above nine steps are the general steps involved in any analysis process, a building-block approach is often used and advocated by experienced analysts. This process is illustrated in Figure 2. A building-block analysis approach begins with basic elements and builds in complexity in a systematic and progressive manner ${ }^{8}$. Such an approach permits each step in the process to be verified and its influence on the overall response determined. First, the problem, objectives, products, resources, schedule, and stakeholders are defined. Second, the structural configuration, loads, materials, boundary/interface conditions, and tools to be used to solve the problem are identified.

Next, analysis models such as finite-element models of the individual components are created. The material modeling procedure, boundary conditions, and other approximations are assigned to the individual component models, and the models are solved and verified for accuracy. The process at the individual component level is an iterative process that is repeated until the results can be verified by comparison to reference solutions or test data. Then, the individual component models are assembled, incorporating component interface conditions, different design configurations, and various load cases. The assembled models are verified, solved, and the results are assessed. The process at the assembly level is also an iterative process that is repeated until confidence in the results can be demonstrated and advocated by the analysis team itself. Finally, the results are presented to external reviewers and the stakeholders, engineering reports are written, and the entire effort is subjected to rigorous peer review. If necessary, the process may be repeated from any of the building-block steps. 


\section{AREAS OF NEGATIVE MARGINS}

During the design of the structure, all possible failure locations and modes are identified. The structure is designed to have sufficient margins of safety for these failure modes at these failure locations. During the preliminary design review (PDR) and critical design review (CDR), the structural analysis is presented by the analysts to a peer group of reviewers based on the status of the design configuration, integrated loads, material characterization, and operating environments. The structural analysts discuss the margins at each anticipated failure location and each of the identified failure modes. Some areas where negative margins are frequently encountered are:

(a) Point loads and load discontinuities

(b) Insufficient mesh density

(c) Stress concentrations and singularities

(d) General modeling issues

(e) Connections

(f) 2D-3D transitions

(g) Boundary conditions

(h) Bolts and bolt modeling

Each of these areas is discussed in the remainder of this section.

\section{Point Loads and Load Discontinuities}

Point loads, concentrated loads, and loads with sharp changes can be easily applied to the finite element models. However, the interpretation of stresses near those areas needs to be well understood before margins can be written at those locations. To illustrate this view point, consider the following two problems from the classical theory of elasticity - the Boussinesq's and punch problems.

Boussinesq's Problem*: Figure 3 shows the problem of a point load $P$ on a half plane. The exact solution was obtained by Boussinesq (see Ref. 9) as

$$
\sigma_{r}=-\frac{2 P}{\pi} \cdot \frac{\cos (\theta)}{r} ; \quad \sigma_{\theta}=0 ; \quad \tau_{r \theta}=0
$$

and

$$
\begin{aligned}
u= & -\frac{2 P}{\pi E} \cos (\theta) \cdot \log (r)-\frac{(1-v) P}{\pi E} \theta \cdot \sin (\theta)+\text { rigid body terms, and } \\
v= & \frac{2 v P}{\pi E} \sin (\theta)+\frac{2 P}{\pi E} \sin (\theta) \cdot \log (r)-\frac{(1-v) P}{\pi E} \theta \cdot \cos (\theta)+\frac{(1-v) P}{\pi E} \sin (\theta) \\
& + \text { rigid body terms }
\end{aligned}
$$

\footnotetext{
* For the two elasticity problems discussed here, the original coordinate system used by Timoshenko and Goodier (Ref. 9) is retained for clarity.
} 
where $\sigma_{r}, \sigma_{\theta}, \tau_{r \theta}$ are the radial, tangential, and shear stresses, respectively, and $u$ and $v$ are displacements in polar coordinates, $(r, \theta)$. This exact solution was obtained by distributing the point load at $r=0$ (see Figure 3 ) by a statically equivalent radial stresses $\sigma_{r}$ such that

$$
\int_{-\pi / 2}^{\pi / 2} \sigma_{r} \cdot r \cdot \cos (\theta) \cdot d \theta=-P
$$

Clearly, the radial stress, Eq. 6, is singular at the point of application of the load $(r=0)$. Furthermore, from Eq. (7) the displacements have a logarithmic singularity at this point as well. Note that the elasticity solution is exact and is valid at every point of the semiinfinite domain except at the point $r=0$.

Numerical solutions to this problem model a large region to simulate the semi-infinite domain and apply the load at the origin. Refinement of mesh density in the region close to the point load will result in non-convergent stresses at the point of application of the load and thus may lead to negative stress margins.

Punch Problem: Figure 4 shows a uniform pressure $p$ on a part of the semi-infinite plane defined by $(-a \leq x \leq a, y=0)$. The exact solution for this problem using an Airy's stress function can be derived by superposition of results in Problems 16 and 18 on pages 146 and 147, respectively, of Reference 9 as

$$
\begin{aligned}
& \sigma_{x}=-\frac{p}{\pi}\left[\arctan \left(\frac{y}{x-a}\right)+\frac{(x-a) \cdot y}{(x-a)^{2}+y^{2}}-\arctan \left(\frac{y}{x+a}\right)-\frac{(x+a) \cdot y}{(x+a)^{2}+y^{2}}\right] \\
& \sigma_{y}=-\frac{p}{\pi}\left[\arctan \left(\frac{y}{x-a}\right)-\frac{(x-a) \cdot y}{(x-a)^{2}+y^{2}}-\arctan \left(\frac{y}{x+a}\right)+\frac{(x+a) \cdot y}{(x+a)^{2}+y^{2}}\right] \\
& \tau_{x y}=-\frac{p}{\pi}\left[\frac{y^{2}}{(x-a)^{2}+y^{2}}-\frac{y^{2}}{(x+a)^{2}+y^{2}}\right]
\end{aligned}
$$

The boundary condition on the shear stress along $y=0$ is $\tau_{y x}=0$. The boundary condition along $y=0$ is exactly satisfied by Eq. (9). However, if the points $( \pm a, 0)$ where the loading is discontinuous are approached along the lines $x= \pm a$, then the shear stress as $y$ approaches zero is given by $\tau_{x y}(-a, y) \rightarrow+p / \pi$ and $\tau_{x y}(+a, y) \rightarrow-p / \pi$ rather than zero as required by the boundary condition.. Therefore at the points $( \pm a, 0)$, $\tau_{x y} \neq \tau_{y x}$ due to load discontinuity. 
Finite element solutions for this punch problem are obtained ${ }^{10}$ and compared with the exact solution in Eq. (9) in reference 10. The problem is reanalyzed and the new results are presented. Using symmetries only one half of the domain is analyzed. The domain was idealized using eight-node plane-strain isoparametric elements. Four mesh models coarse, medium, fine, and finer - were used. The medium mesh was obtained by subdividing each element of the coarse mesh into four elements. Similarly, subdivisions were used to develop fine and finer mesh models. The four idealizations are presented in Figure 5.

Figure 6 shows the normalized shear stress distribution, $\tau_{y x}$ on the line $y=0$, which should be zero. The finite element shear stress results for the four meshes are approximately zero except in the neighborhood of point A. Very near point A the shear stress $\tau_{y x}$ had relatively larger positive and negative values. However, for all four meshes the non-zero values were confined to two elements on either side of point $A$. The integral of the shear stress $\int_{0}^{10 a} \tau_{y x} \cdot d x$ on the $y=0$ line was nearly zero for all mesh refinements.

Figure 7 presents the normalized shear stress distribution $\tau_{x y}$ on the line $x=a$ for $0 \leq y \leq a$. The finite element solution with the four meshes agreed very well with the exact solution except in the immediate neighborhood of point A. Again, the region of disagreement was confined to the two elements nearest the discontinuity. Numerical integration revealed that the equilibrium condition $\int_{0}^{10 a}\left(\tau_{x y}\right)_{x=a} \cdot d y=p a$ is satisfied approximately for all idealizations.

The exact solutions outlined here were obtained using the Airy's stress functions that was developed from the equilibrium equations with $\tau_{x y}=\tau_{y x}$ at all interior points of the region. The only condition imposed on the solution at the boundary was that it satisfies the applied boundary conditions - symmetry of the stress tensor derived from the Airy's stress function is not required at boundaries.

In both the problems discussed, the behavior of the exact solution suggests that the loading as shown in Figures 3 and 4 is not physically possible ${ }^{9}$. A concentrated load (see Figure 3) cannot be applied physically at a single point, rather the load needs be distributed over an area. Similarly, in the punch problem (see Figure 4) loads cannot be applied with a sharp discontinuity at points $x= \pm a$. The loads need to ramp down from $p$ to zero over some length. When the loading is replaced by physically admissible loading, the inconsistencies seen in the elasticity solutions do vanish ${ }^{9}$.

These examples suggest that the FE solutions are accurate everywhere except very near the load discontinuity. The inaccuracy appears to be limited to two elements. Such regions can be made small by progressive mesh refinements. 


\section{Insufficient Mesh Density}

In most aerospace practice, full-vehicle finite element models are available as these models are extensively used in loads evaluations and system integration. However, the tendency is to use these loads models to evaluate margins even though there is not enough fidelity to capture the local stress states. When stress results from such global models are used for margin calculations, the resulting negative stress margins may not be real. For example, global FE results of an entire upper stage simulator (USS) segment of the Ares I-X vehicle generally over predicted the peak stress levels in secondary structures due to local modeling issues, point loads, and constraints. From a buildingblock perspective, loads models have entirely different objectives than stress models. Modeling approximations in loads models, even detailed loads models that may result in negative stress margins include:

- FE approximations of local configurations such as curved boundaries approximated by a few straight segments, thick regions approximated by shell elements, and long members approximated as beam elements.

- Application of 'point' loads through the intersection of different element types (beam tied to solids or shells)

- Point reactions from rigid body element (RBE) models tied to a limited number of nodal points that 'push' as well as 'pull' locally in a bolt hole

- Limited mesh refinement to represent local stress gradients within the structure.

The use of loads models to determine internal loads that are then imposed on separate local stress models is the preferred approach. Local models with satisfactory fidelity need to be developed to delineate the stress field and only then can the margin of safety be evaluated effectively.

\section{Stress Concentrations and Singularities}

Aerospace structural components inherently have stress raisers such as holes, free edges, stringers, thickness changes, etc. Stresses near the edge of holes can be three times the remote applied stress in isotropic materials like metals. Composites, on the other hand, are highly sensitive to notches. Stress concentrations factors higher than a value 10 are possible for some notches in unidirectional composites. Also, in composites, large inplane stress gradients develop near geometric and material discontinuities. These large in-plane stress gradients lead to out-of-plane, through-the-thickness, or interlaminar stresses. (Interlaminar stresses at a straight free edge in simple $[0 / 90]_{\mathrm{S}}$ and $[+45 /-45]_{\mathrm{S}}$ laminates were first discussed in a classic paper by Pipes and Pagano in 1970 in reference 11). Because composites have low through-the-thickness strength, delaminations can initiate and grow. Delaminations degrade the strength of the composite laminate and can lead to failure. Figure 8 shows some typical configurations susceptible to interlmainar stresses where delaminations initiate because of geometric and material discontinuities. Configurations shown in this figure are quite frequently encountered in aerospace structures and structural components. While software tools are now available to rapidly model complex configurations shown in this figure, knowledge 
of isotropic and anisotropic elasticity is needed to correctly interpret the finite element stress results.

Stresses grow without bound at stress singularities. Also, near singular points large gradients of stresses are encountered. The strength of the singularity dictates the gradient of the stresses in these regions. Figure 9 shows the singularity problems discussed by Williams ${ }^{12}$ and Hein and Erdogan ${ }^{13}$. Figure 10 presents the finite element model of a plate with one edge clamped along $y=0$ and the other edge (along the $x=0$ line ) with a stress free condition. Williams ${ }^{12}$ showed that a stress singularity exists at $r=0$ for this case. He also considered a problem of a crack, Figure 9 (b), where the crack faces are stress free,

$$
\text { on } \theta= \pm \pi, \sigma_{\theta}=\tau_{r \theta}=0 \text {. }
$$

He showed that the square root singularity exists at the crack tip, $r=0$.

The stresses on any radial line from the singular point A (or A') have the form (see Ref. 12)

$$
\{\sigma\}=\{\mathrm{C}\} r^{-\alpha}+\mathrm{O}\left(r^{-\alpha+1}\right)
$$

where $\{\sigma\}=\left\{\sigma_{r}, \sigma_{\theta}, \tau_{r \theta}\right\},\{C\}$ is a vector of constants, $r$ is the radial distance from point A and $O\left(r^{-\alpha+1}\right)$ represents terms of the order of $r^{-\alpha+1}$ and higher. The exponent $\alpha$ is the singularity power. For the case of a fixed-free plate, plane strain conditions, and $v=0.3$, the $\alpha$ has a value of 0.289. As the point $\mathrm{A}$ is approached along the bond line, $y=0$, the shear stress $\tau_{y x}$ will be singular with $\alpha=0.289$. But as the point $\mathrm{A}$ is approached along the free edge, $x=0$, the shear stress $\tau_{x y}$ has a zero value. Therefore, the shear stress and its complement are unequal at the singular point. For a crack problem, the singularity is stronger with a value $\alpha=0.5$.

Figure 11 presents the normalized shear stress along line, $y=0$, obtained with the coarse, medium, fine (see reference 10) and finer meshes. Because of the singularity, the shear stress has a steep gradient very near $(x / a)=0$ and does not show convergence of the peak shear stresses with mesh refinement. Figure 12 shows normalized shear stress distribution along the $x=0$ line. As expected, the shear stress was nearly zero all along the free edge and is non-zero only near the singular point. As in the load discontinuity case, the regions of non-zero values are confined to two element thicknesses. Numerical integration showed that the integral of the shear stress

$\int_{0}^{8 a}\left(\tau_{x y}\right)_{x=0} \cdot d y$

is nearly zero for all mesh refinements.

This example suggests that the finite element solutions are accurate everywhere except 
very near a stress singularity. The stresses do not show convergence in these small regions. Judicious choices need to be exercised in interpreting the FE results near stress discontinuities and singularities.

Numerical methods try to delineate the stress field in the regions of load discontinuities and stress singularities to the best of their ability. However, the analysts should recognize the load discontinuities and stress singularities, adopt appropriate modeling and analysis strategy, and interpret the results appropriately. This is where the knowledge of elasticity and exact solutions would be very insightful.

\section{General Modeling Issues}

Inherited Models: An inherited model is a model that is passed from the analyst that developed the model to other analysts - perhaps sitting next to each other, perhaps different companies, perhaps decades apart. A common assumption is that the inherited FE model was verified and accurate as received, which is generally true for the purpose of the model. However, often times a model developed for one purpose is enlisted for other purposes for which it was not verified. A common theme observed during some PDRs and CDRs is that the analysts were pressed for time and independent status checks of the inherited FE models are not always performed, but rather are assumed to have been performed during the model's initial development. Another common assumption is that all engineering change orders made to the hardware during fabrication and assembly are assumed to have been implemented within the FE models. It is not uncommon that the analysts performing the current assessments are not the analysts that developed the initial FE models.

Thorough evaluation of the influence of the loads on stress and dynamic performance requires time (i.e., to get familiarized and resolve potential problems). Model changes may be required as a result of changes in the loads or changes noted between the hardware and the FE model. Whether an existing FE model does or does not indicate a problem does not mean that the model is correct, especially when independent model checking is not performed as part of the initial model development process. Running the previous models with the new loads and getting positive margins is good and necessary, but not sufficient.

Modeling Issues: FE approximations of local configurations such as curved boundaries approximated by a few straight segments, thick regions approximated with thin shell elements, and long members approximated as general elastic beams, are widely used in loads models. Stress analysts need to investigate and determine if this type of local modeling is adequate for their stress models. Modeling issues related to how point loads and constraints are simulated in the models determine whether high localized stress values are due to 'artifacts' of the modeling or that a true local stress gradient exists. The ability to apply proper boundary conditions in some software is limited, and as a result many parts of the model can be over-constrained and do not develop appropriate stress fields for all the load cases under consideration. This may result in unconservative analysis results. 
In a few analyses reviewed, many areas of high stress were considered analysis artifacts and dismissed by the analysts. In these regions, the stress field was more pervasive than localized. The analysts need to pay special attention to these regions and determine if their hypothesis is true. Material nonlinear analyses can be used to assess the validity of the peak values in the stress field and exonerate the design.

In general, modeling issues arise when an analysis model for one purpose (e.g., internal loads determination) is taken and applied for other purposes outside of the original intent (e.g., local stress predictions). In most cases, such decisions are made for expediency to meet schedule demands or due to limited resources; however, often more time is taken to explain and rectify unexpected results than developing the appropriate model would require from the start.

Incorrect elements: The use of linear tetrahedral or linear triangular elements needs to be limited and should not be used in regions with local gradients or bending. In some cases, rod elements are used to simulate an axial member or stiffener, only to later realize that axial and bending loads are to be carried by that structural member. When such elements are used and local gradients are not indicated, the analyst needs to verify that the FE model is actually correct in not predicting a local gradient or whether the FE model itself is the cause of no local gradient. This process can be difficult to assess unless other models can be developed and analyzed following a building-block approach for modeling and analysis. Regions that exhibit local gradients or bending may need to be re-modeled using linear quadrilateral, hexahedral elements, quadratic triangular or tetrahedral elements to account for such local effects. Re-modeling local regions using quadrilateral and hexahedral elements or quadratic triangular and tetrahedral elements should be considered as an alternative. The analysts who are not familiar with the FE methodology need to invest time in studying the performance of these elements. Many excellent text books (e.g., see Refs. 14-16) discuss these issues.

\section{Connections}

Structures such as ladders, platforms, piping connections and hangers, etc. are encountered in rocket structures and are termed as secondary structures. When such secondary structures are connected to primary structures, the interface conditions need special attention. Bolted flanges of the pipe hangers and brackets also need special attention. Simulating the interaction of different attached components is a challenge. The assembly process for bolted flanges of the pipe hangers and brackets needs to be verified to determine whether the bolted flanges are in contact initially or whether the bolt preload 'draws' the two flanges together within the FE models. If the former is true, then including the bolt preload is needed only in the bolt margins. However, if the latter is true, then the bolt preload may be introducing local bending in the flange that would influence the flange/bracket margins. If these flanges are modeled using shell elements and they are initially in contact, then constraints should be in the FE model to maintain the relative position of the two flanges with respect to each other. 
Approximating one component using assumed boundary conditions applied to an adjacent component requires an assessment of relative stiffnesses. The use of 'clamped' or 'fixed' conditions is probably not the actual hardware condition. Inclusion of a component's stiffness through the use of NASTRAN type DMIG-like terms (Direct Matrix Input at Grids as in a substructure interface modeling approach) or by direct inclusion of all components should be carefully assessed.

These connection regions commonly exhibit negative margins, and the negative margins are typically due to the inadequate representation of the component interface conditions in the finite element model.

\section{D - 3D Transitions}

There are several instances where the 2D-3D transitions are used: to affect local refinements in areas where higher fidelity stresses are needed, to affect transitions from plate/shell modeling to 3D modeling, or at connections between secondary and primary structures. Most often, along the 2D and 3D interface, the degrees of freedom are not compatible due to different kinematic assumptions. The corresponding degrees of freedom are subjected to multi-point constraints to affect the transition. Alternatively, some special equations are written to relate the 2D degrees of freedom (dof) to the $3 \mathrm{D}$ dof. The transition regions are generally a source of negative margins and usually these negative margins are fictitious. However, analysts need to take extra precautions to examine the stress states to determine that the negative margins are in fact real.

\section{Boundary Conditions}

Analysis tools need to be capable of defining arbitrary boundary conditions and constraints, perhaps using different local coordinate systems, in order to restrain the structure properly. In a recent assessment, the ability to apply proper boundary conditions to a complex structural model using the chosen analysis software was limited. As such, several parts of the structural model were over-constrained and did not develop appropriate stress fields, resulting in unconservative analysis results and subsequent negative margins observed in test. When such situations develop, the use of classical hand analyses to verify the finite element results is critical. Careful hand analyses can be effectively used to interrogate the FE analysis results. An example is presented in the appendix to demonstrate how a hand analysis can be used to verify finite element results.

In the same assessment that was discussed above, many areas of high stress were considered analysis artifacts. Material nonlinear analyses should be used as an appropriate method to assess the validity of the peak stress field. The lesson that is learned from this analysis is that some of the software tools that are in use are valuable for design iterations but are not suitable for evaluating final margins of safety. Margins need to be evaluated using detailed analysis models with appropriate boundary conditions, and it may be necessary to switch from a design tool to a more general analysis tool. Understanding the tools being used and their limitations is fundamental. 


\section{Bolts and Bolt Modeling}

Bolts and joints are complex structural configurations. There is no universally accepted process for modeling bolts in the joints. Bolt and joint modeling needs to be examined in any FE model to ensure all appropriate load paths are considered for the appropriate margin calculations.

Typically, bolts, nuts, and washers are not explicitly represented in the finite element models, but rather their influence is simulated. Some typical modeling strategies for bolted structures include: simply smearing the thickness of the fastened parts together and ignoring the discrete bolts; introducing discrete constraints or beam element at every fastener location and ignoring features of the bolt; including the fastener as one or more beams to simulate the bolt shank and with sets of constraints to simulate the bolt head and nut (i.e., beam and spider approach) usually coupled with an explicit model of the fastener hole; and detailed 3D model of the bolt head, shank, washer(s), and nut including contact and friction. In each strategy, the analyst needs to consider the modeling of the parts being fastened together as well as the fastener modeling details. If the fastener is installed with a specified preload, then analysis procedures to impose the preload need to be developed (e.g., use of thermal loading to obtain the preload, or some special feature of the analysis tool).

The most common approach used to model mechanically connected parts is to use the beam and spider approach in which the bolt shank is represented as one-dimensional linear elastic beams, while the bolt head, washer(s), and nut are represented as sets of kinematic coupling constraints. These kinematic coupling constraints extend radially outward from the bolt shank centerline to nodes located a specified distance around the bolt hole and on the non-mating flange surfaces to simulate the bearing load. The nodes defined in these sets of constraints then respond as a rigid plane that can translate and rotate based on the beam element response for the bolt shank. The beam element is allowed to bend; however, contact with the edges of the bolt hole is not simulated. Hence, this type of constraint can represent the effects of bolt bending on the local stress state in the flange near the washer. The washer-bearing-surface modeling assumptions are discussed in Reference 17 and 18. Most experienced analysts use ring-type modeling around the bolt hole and discard the stresses in the two rings that are closest to the hole, as the kinematic coupling spider constraints develop spurious stress results in those rings.

In critical bolted connections, the bolt head, bolt shank, washer(s), and the nut are explicitly modeled by 3D elements along with the explicit modeling of the bolt holes. Complexities associated with contact, friction, interference fits, and preload application need to be addressed in detail. The beam-and-spider approach and the explicit 3D approach usually provide the same overall response when the bolted members have flat parallel surfaces. However, in cases where the non-mated flange surfaces are inclined, the FE results are sensitive to these local modeling assumptions and need to be examined.

In these models, the boundary conditions in the axial direction (along the bolt) may be required to account for sharing the applied axial load (along the bolt axis), but shear 
boundary conditions may only be correct for a subset of bolts due to clearances and/or ovality of holes. Load sharing needs to be examined when margins are to be assessed. When bolting dissimilar materials together, the effect of different coefficients of thermal expansion values should be included in the shear loading of the joint.

Shear loading in bolted connections requires review to assess questions related to: assembly clearances and slotted holes; load sharing for a series or row of bolts; double shear for joints; and shear loading due to differential thermal expansion.

In some cases, the negative margins on yield need to be resolved by studying the sensitivity to the preload generated by the applied bolt torque. Assembly, dis-assembly, and re-assembly may also be a condition to be examined. For bolts that are 're-torqued' the procedure for the re-applying the bolt torque needs to be defined (for example, will the affected bolts be completely loosened and then re-torqued?).

Hand-calculations for bolt bearing and bolt performance using the forces from the FE model need to be used and compared rather than using the stress value from the FE analysis directly. The stresses from the FE analysis may be artificially high due to concentrated or point loading. Bolt forces can be extracted from the FE results and hand calculations of the local response performed. Analysts need to determine the number of structural members bolted together (e.g., single vs. double lap joint) and assess any load sharing simulated by the FE model.

\section{SOME SUGGESTIONS FOR ANALYSTS}

A few pieces of advice are offered to early-career analysts.

Verification: Frequently the analysts performing the current evaluations are not the analysts who developed the initial FE models. The analysts are usually hard pressed for time. As such, independent status checks of the inherited FE models are not always performed. The analysts who received the models assume that all the verification checks have been performed and the model is accurate as received. The analysts should take the time to perform all the verification checks on the received model. The analysts should also ensure that the analysis model is understood and consistent with the analysis requirements for which the model is being used.

FOS: The analysts should ensure the appropriate factors of safety are utilized in the analysis.

Changes to Hardware: Model changes may be required as a result of changes in the loads or changes noted between the hardware and the FE model. Given that an existing FE model does or does not indicate a problem does not mean that the model is correct, especially when independent model checking is not performed as part of the initial model development process. Running the previous models with the new loads and getting positive margins is good and necessary, but not sufficient. The analysts should ensure 
that the model represents the as-fabricated hardware and that all applicable engineering change orders are incorporated.

Traceabiltiy: All material data, component configurations, and loads need to have traceability to program requirements. The consistency of these features should be established through documented cross-checking of the analysis models with the final drawings and engineering change orders. The analysts should ensure traceability to design requirements or other source documents for all input parameters critical to the analysis and margin calculations.

Allowables: The analysts should ensure the consistency of allowable values with traceability to materials testing reports.

Buckling vs Strength: Most thin wall structures are much more critical in compression than tension due to susceptibility to buckling and crippling. Parts that appear to be prone to buckling failures need to be explicitly identified. For these cases, margins should be written against the buckling allowable and not the yield stress in compression. In some cases post-buckling behavior needs to be analyzed to determine the full capability of the structure.

Stress vs Failure Predicitons: Both stress and failure predictions can be attempted using classical or FE methods. While there have been many accurate stress analyses using classical or FE methods, fewer successes are reported with failure predicitons. The analysts should invest time and effort in understanding, modeling, and prediction of failure.

Hand calculations: There are many problems that can be solved using classical methods utilizing beam, plates and shell theories. If there are classical solutions available for a reasonable model of the structure, those classical solutions should be used. Aerospace companies have analysis manuals that capture application of these methods to aerospace structures. The use of classical hand analyses to verify the finite element results is critical. The analysts should develop hand analyses skills and tools to interrogate the results of their FEA. An example of how a hand calculation can be performed to evaluate a FE result is presented in the appendix.

Elements: The analysts should ensure the modeling is performed with elements that perform well within reasonable ranges of distortions. Instead of depending on the documentation supplied by the software tool developers, the analysts should develop their own experience database. They should experiment with various element types and mesh configurations, apply them to well known classical problems, and study their performance. Examples of such problems can be found in reference 19 by MacNeal and Harder. 


\section{CONCLUDING REMARKS}

Aerospace structures are complex,high performance structures. There is an increasing reliance on modeling and simulation to verify, qualify, and certify designs. Advances in reliable and efficient computing and modeling tools are enabling analysts to model complex three dimensional (3D) configurations and perform analysis rapidly. Very frequently the analysts performing these analyses would blindly accept results that the software provides and use those results to report negative margins of safety. Some of these negative margins are not real but are artifacts of the modeling and are sometimes due to idiosyncrasies of the analysis software. This paper is aimed at raising awareness to question the negative margins and suggest that analysts need to pay attention and evaluate those results carefully before accepting the negative margins.

Knowledge of the behavior of structures and the theory of elasticity, the ability to formulate an estimate of expected results before they are obtained, the awareness of consequences of modeling assumptions, etc. are essential to interpret the numerical results. Only then can one ascertain if the observed negative margins are in fact real. The areas where negative margins are frequently encountered are point loads and load discontinuities, locations of stress singularities, large gradient areas with insufficient mesh density, areas with modeling issues and modeling errors, connections and interfaces, 2D-3D transitions, and boundary conditions. For early-career analysts, the authors hope that this paper may serve as motivation for learning and/or relearning the theory of elasticity, the theory behind the finite element method, and other closed-form solution procedures. For seasoned analysts, the authors hope that this paper may serve as motivation for mentoring their junior colleagues.

\section{Appendix A}

\section{An Example Problem}

This appendix presents an example problem that depicts the importance of the role of the analyst in defining, simplifying, researching, and verifying analyses.

The problem context is the Mars Science Laboratory Descent Stage. In the past, propulsion system plumbing used relatively flexible 0.25 in. diameter lines. These lines were relatively compliant compared to the primary structure. In the case of the Descent Stage, the propulsion system lines were comprised of diameters up to 1". These lines were comparable in stiffness to the primary structure and therefore, like primary structure, knowledge of the loading in propulsion lines would need to be developed and margins of safety computed. For the first time at JPL, the entire propulsion system layout was modeled together as part of the primary structure (see Figure 13). 
The problem statement was to (a) develop practical analytical models to determine element loads for the propulsion system lines and (b) to develop a method for determining the state of stress in the propulsion lines. Figure 13 depicts the complexity of the propulsion system plumbing with compound bend angles and thermal isolating support bracketry. To understand the behavior of bends in propulsion lines, a simplified test configuration shown in Figure 14 was developed. This test configuration was amenable to hand analyses and also could be easily tested in the laboratory using an Instron testing machine.

A literature research was conducted on the behavior of bent tubes. References 20 and 21 suggested that the stiffness and stress of bent tubes does not adhere to the standard linear beam element formulations. The literature showed that the stiffness of bent tubes is affected by the degree of curvature of the bend as well as the magnitude of pressurization. The lines can be seen to provide redundant load path to the primary structure and undergo compatible displacements. Good approximations of stiffness are required to provide accurate estimate of loads in the propulsion lines as part of the Descent Stage structure.

To understand the stiffness characteristics and the stress under load of the bent strut, the test configuration was modeled with three different idealizations: a linear beam element, a shell, and a $\mathrm{CBEND}^{3}$ /beam model. Note that the CBEND/beam model uses CBEND elements in the bends of the propulsion line and beam elements in the straight sections. The results obtained by the use of these models then were compared to test data. Figure 15 shows the results of the analyses and test. The analysis shows that a bent tube exhibits nonlinearity in stiffness both in compression and tension. The analyst's goal is to develop bounding loads and therefore, it is sufficient to use a model that provides reasonable tension stiffness. The all-beam model is more than 2.5 times stiffer than the measured tension stiffness. In contrast, the all-shell model and the CBEND/beam model yielded reasonable agreement to the tension stiffness. For both the loads and /stress models of the propulsion layout, the CBEND elements were chosen since it would be impractical to develop shell models for the entire layout of the propulsion system tubing.

The literature search also suggested that the longitudinal stress in the tube requires correction in the area of the bends. Maximum longitudinal stress in a beam element is normally computed using combined tension - bending formula, $(\mathrm{P} / \mathrm{A}+\mathrm{Mc} / \mathrm{I})$. For the test strut shown in Figure 15, this value was $13 \mathrm{ksi}$ when linear beam element model was used. The shell model was exercised to the same level of applied load. The peak longitudinal stress was $21 \mathrm{ksi}$ (see Figure 16). The ovalization of the tubing in the bend at the location of maximum moment is depicted in Figure 16. This effect intensifies the nominal stress magnitude.

Compression tests of the bent tube were instrumented as shown in Figure 14. The measured strains were used to evaluate the peak longitudinal stress and a value of $21 \mathrm{ksi}$ was obtained. Thus the stress magnitude and the location of the peak longitudinal stress were validated by testing.

\footnotetext{
${ }^{3}$ CBEND is a NASTRAN linear beam element that represents a bent beam.
} 
Classical hand analyses for stress can be performed to verify the FE results. This calculation can be performed as follows. The literature research shows that a stress intensification factor, the Rodabaugh factor, should be applied to adjust the peak longitudinal stress. This factor requires only geometric parameters to correct the nominal stress. The factor is evaluated as follows ${ }^{20}$ :

If $\mathrm{R}=$ Radius of curvature of bend, $\mathrm{r}=$ radius of the tube, and $\mathrm{t}=$ thickness of the tube wall, then $\mathrm{h}=\mathrm{t} \times \mathrm{R} / \mathrm{r}^{2}=.035 \times 3 / .5^{\wedge} 2=.42 \mathrm{in}$. and the Rodabaugh factor is $\mathrm{I}_{\mathrm{R}}=.9 /\left(\mathrm{h}^{2 / 3}\right)$ $=1.6$. The peak stress can be computed as $=1.6 \times 13 \mathrm{ksi}=21 \mathrm{ksi}$

Thus, the tension-bending stress formula adjusted by the Rodabaugh factor agrees well with test and with the results of the more complicated shell finite element model. The method for determining the state of stress for the MSL Descent Stage propulsion lines is to simply use the tension-bending stress formula with the Rodabaugh stress intensification factor. Using this technique greatly simplifies the modeling effort. $\mathrm{CBEND/beam}$ element forces are used to compute stresses and the need to develop full shell models is avoided.

In summary, literature research was pivotal in understanding the behavior of bent tubes. Testing combined with different modeling approaches was used select the proper modeling idealization required for accurate loads prediction. In this real flight example of loads determination, 2000 propulsion line elements were used. The element types selected were the CBEND and beam elements. The stress was computed from the element force results using the tension-bending formula adjusted by the Rodabaugh Stress Intensification factor. The methodology selected was practical, easily implemented, and accurate. This example problem demonstrates how an analyst can define, simplify, research, and verify models and methods.

\section{References}

${ }^{1}$ Anonymous, NASGRO 6 Reference Manual (2010), Fracture Mechanics and Fatigue Crack Growth Analysis Software, NASA, South West Research Institute, Version 6.0 2010, (www.nasgro.swri.org - accessed March 4, 2011).

${ }^{2}$ Anonymous, AFGRO, AFGROW Users Guide and Technical Manual (2010), AFRLVA-WP-TR-2002, Version 5.01.05.16, J. A. Harter, Air Vehicles Directorate, 2790 D Street, Ste 504, Air Force Research Laboratory, WPAFB OH 45433-7542.

${ }^{3}$ Bruhn, E. F., Analysis and Design of Flight Vehicle Structures, Jacobs Publishing, Inc., 1973.

${ }^{4}$ Anonymous, Astronautics Structures Manual - Volume 1, NASA TMX-73305, August 1975. 
${ }^{5}$ Anonymous, Astronautics Structures Manual - Volume 2, NASA TMX-73306, August 1975.

${ }^{6}$ Anonymous, Astronautics Structures Manual - Volume 3, NASA TMX-73307, August 1975.

${ }^{7}$ Mohaghegh, M., AIAA Short Course "Design of Aircraft Structures", 2011 SDM

Conference, Denver, Colorado, April 2-3, 2011.

${ }^{8}$ Raju, I. S., Knight, N. F., Jr., Song, K. C., and Phillips, D. R, "Fracture mechanics analyses of the slip-side joggle regions of wing-leading-edge panels", Frattura ed Integrita Strutturale, Vol. 15, pp. 35-49, 2011. Also available as NASA TM-2011216878 , January 2011.

${ }^{9}$ Timoshenko, S. P. and Goodier, J. N., Theory of Elasticity, McGraw-Hill Book Company, 1970.

${ }^{10}$ Raju, I. S., Whitcomb, J. D., and Goree, J. G., "A new look at numerical analyses of free-edge stresses in composite laminates". NASA TP-1751, December 1980.

${ }^{11}$ Pipes, R. B., and Pagano, N. J, "Interlaminar stresses in composite laminates under uniform axial extension”, Journal of Composite Materials, Vol. 4, October 1970, pp. 538-548.

${ }^{12}$ Williams, M. L., "Stress singularities resulting form various boundary conditions in angular corners of plates in extension," Journal of Applied Mechanics, Trans. of ASME, December 1952, pp. 526-528.

${ }^{13}$ Hein, V. L. and Erdogan, F., "Stress singularities in a two-material wedge," International Journal of Fracture Mechanics, Vol. 7, No. 3, September 1971, pp. 317330 .

${ }^{14}$ Cook, R. D., Malkus, D. S., and Plesha, M. E., Concepts and Applications of Finite Element Analysis, Third Edition, John Wiley \& Sons, 1989.

${ }^{15}$ Zienkiewicz, O. C. and Taylor, R. L., The Finite Element Method, Vols. 1 and 2, Fourth Edition, McGraw Hill Publishers, 1989.

${ }^{16}$ MacNeal, R. H., Finite Elements: Their Design and Performance, Marcel Dekker, Inc., 1994.

${ }^{17}$ Knight, N. F., Jr., Phillips, D. R., and Raju, I. S., “ Simulating Structural Response of a Preloaded Bolted Joint", Paper presented at the $49^{\text {th }}$ AIAA SDM conference, Schaumburg, Illinois, AIAA Paper No. 2008-1842, April 2008. 
${ }^{18}$ Mohaghegh, M., Tension Analysis of Stiffened Aircraft Structures, AIAA- 85-795, 26th SDM Conference, Williamsburg, Virginia, 1985.

${ }^{19}$ MacNeal, R. H., and Harder, R. L., "A proposed standard set of problems to test finite element accuracy", Finite Elements in Analysis and Design, Vol. 1, 1985, pp. 3-20.

${ }^{20}$ Rodabaugh, E.C. and George, H.H., 'Effect of Internal Pressure on Flexibility and Stress-Intensification Factors of Curved Pipe or Welding Elbows', Transactions of the ASME May 1957, pp 939-948.

${ }^{21}$ Markl, A.R.C., 'Piping-Flexibility Analysis', Transactions of the ASME February 1965, pp 127-149

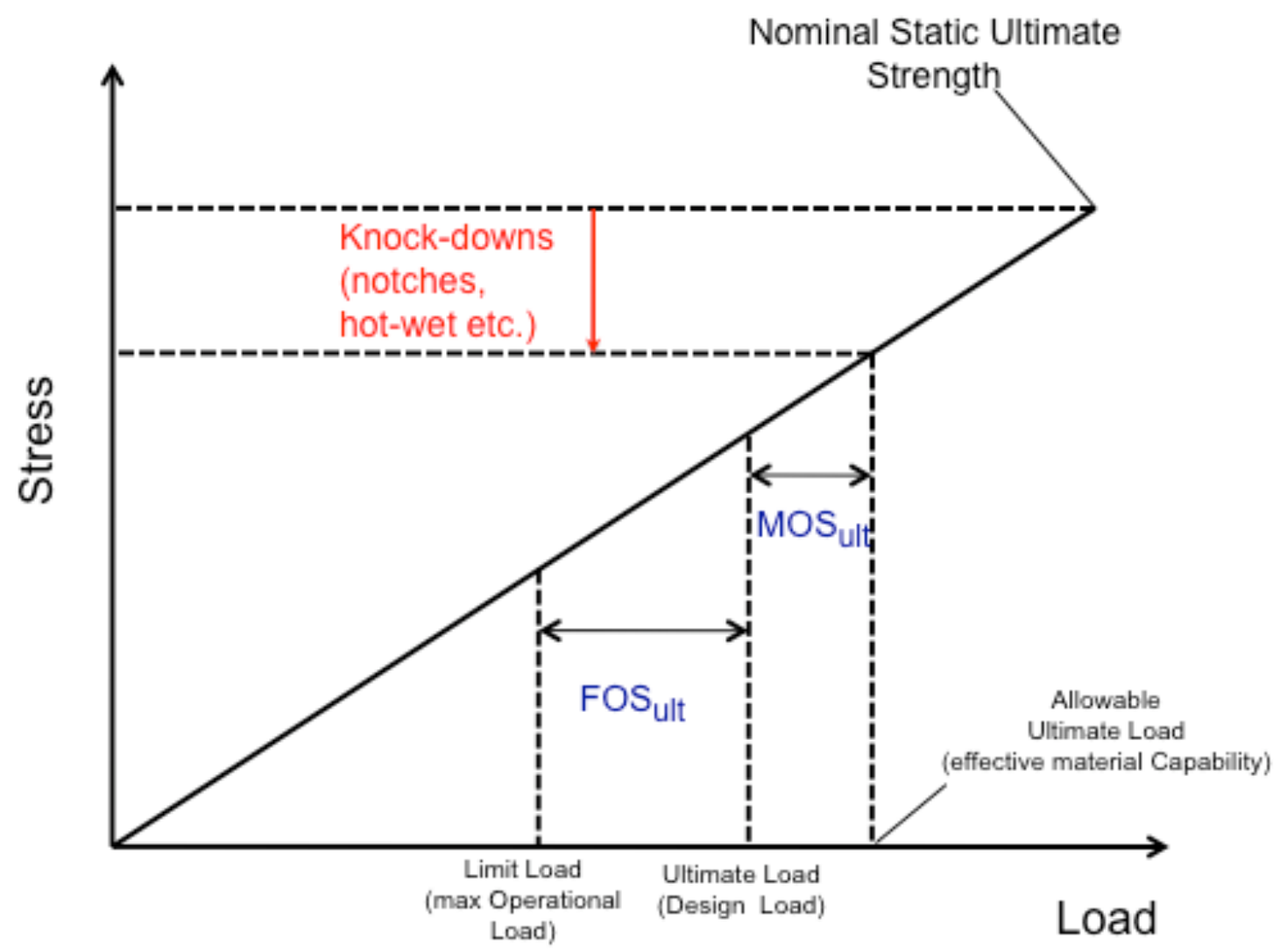

Figure 1. Relationship between factor of safety (FOS) and margin of safety (MOS) 


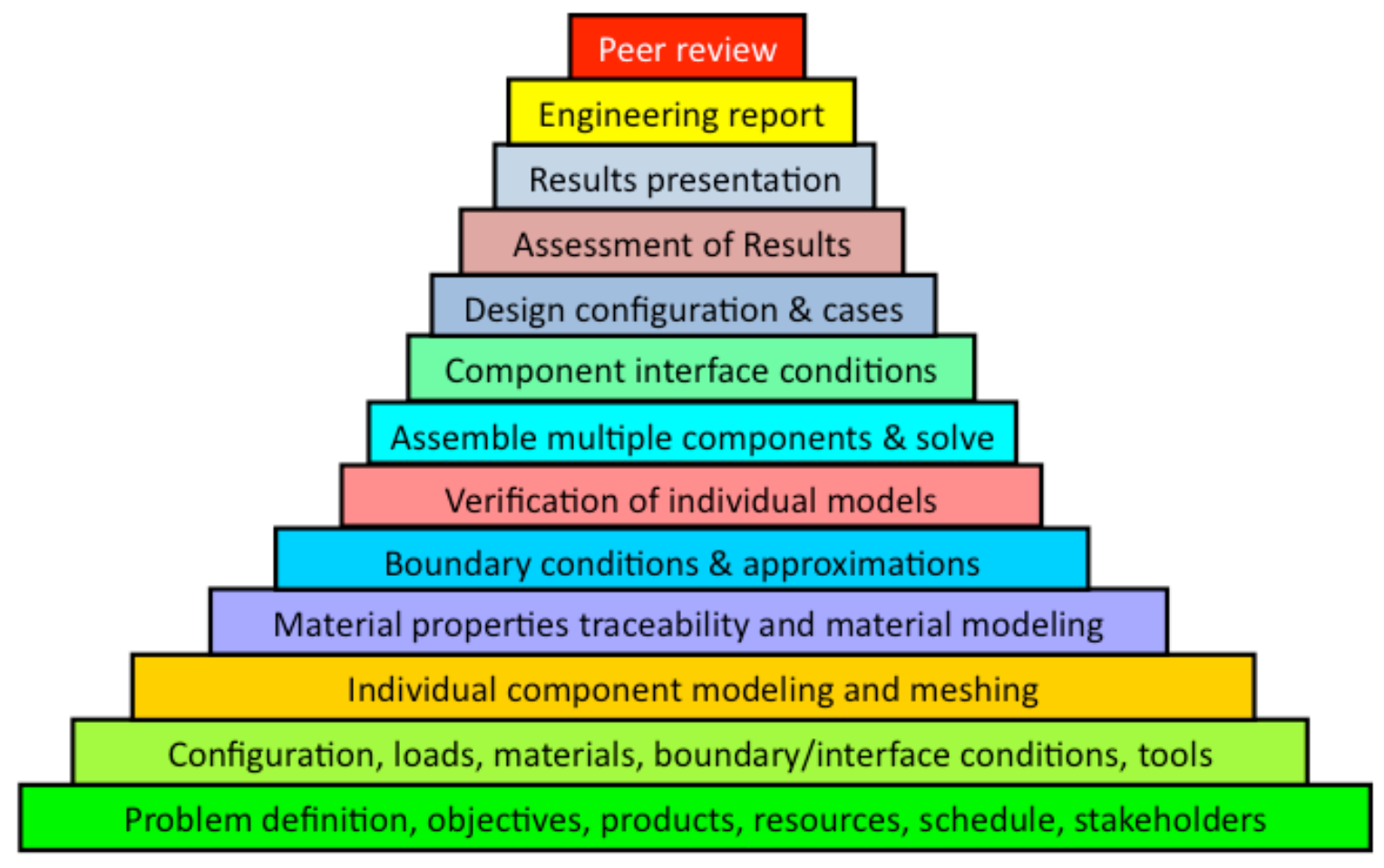

Figure 2. Building Block Approach

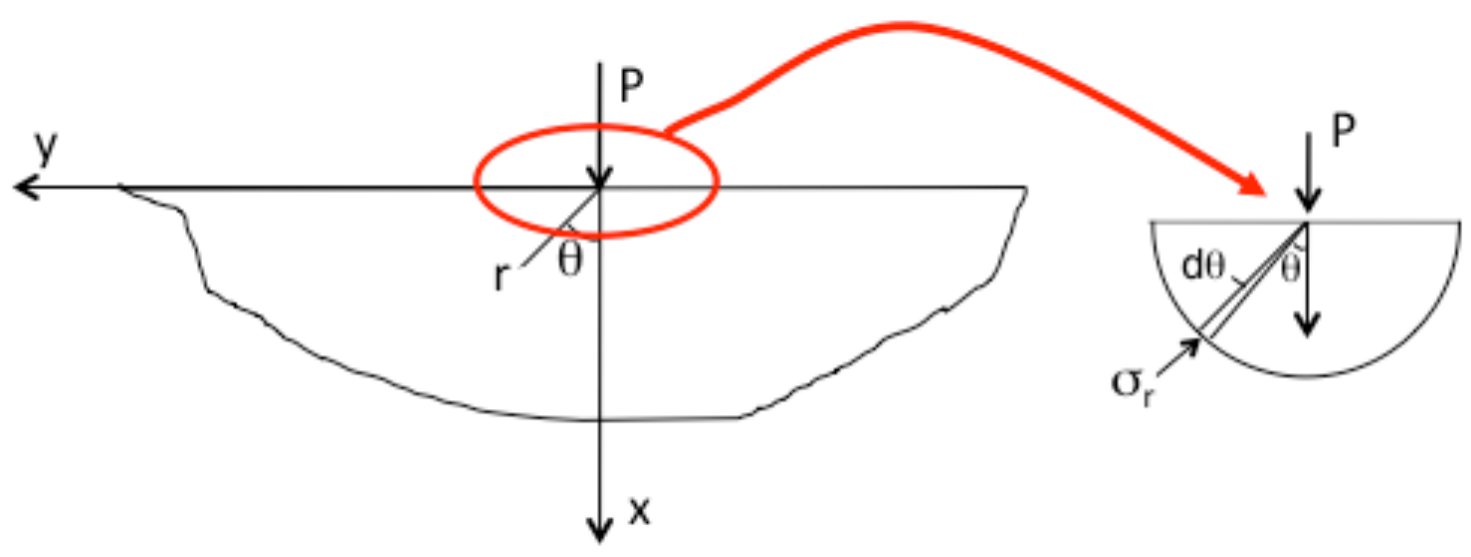

Figure 3. Point load on a semi-infinite plane 


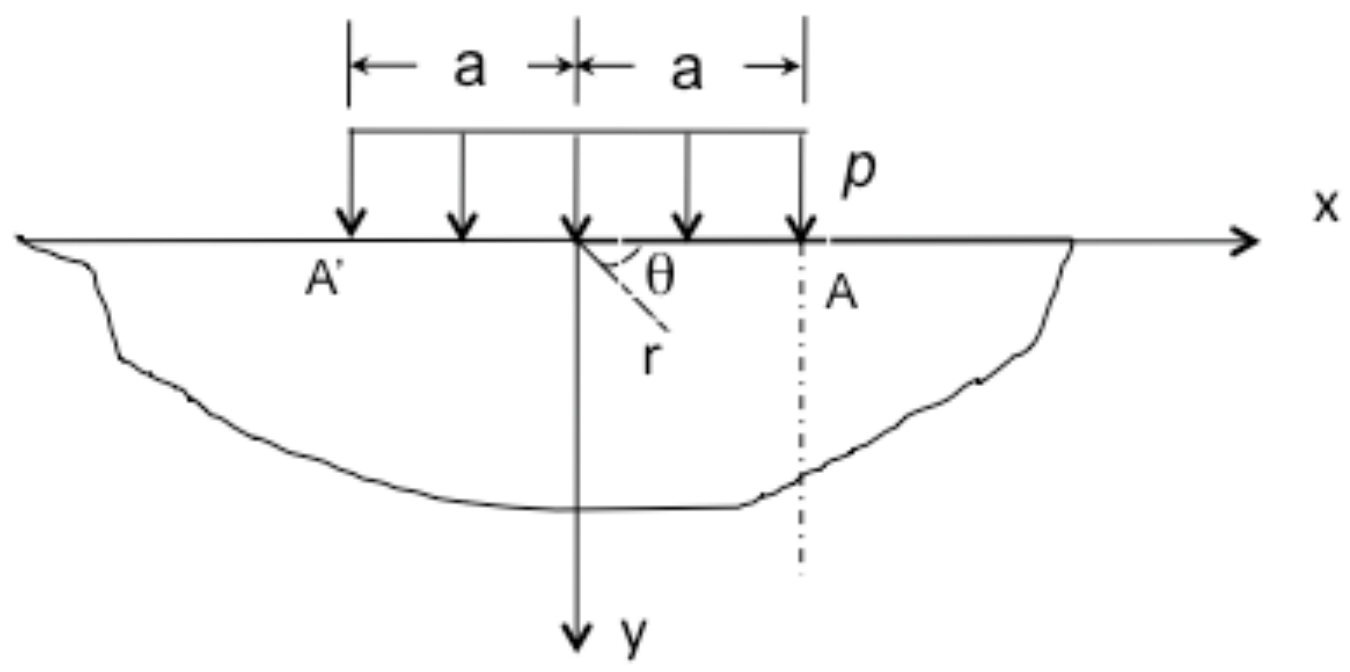

Figure 4. Distributed load on a semi-infinite plane - the punch problem

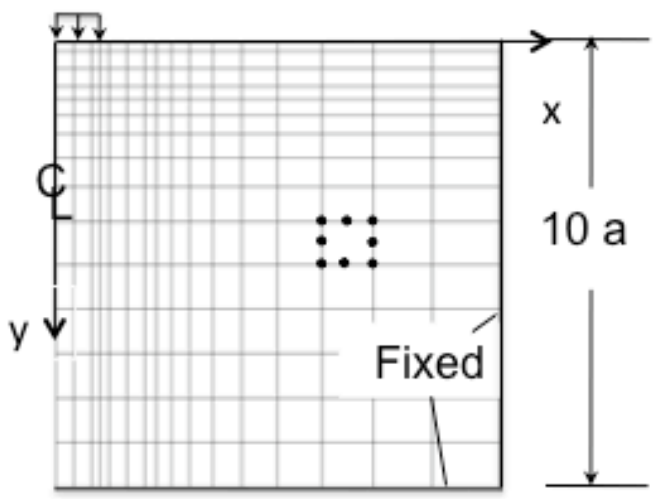

10 a

Coarse mesh

(Elements: 240 , Nodes: 783)

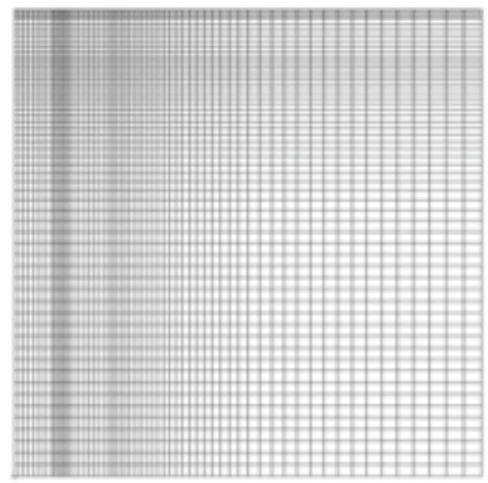

Fine mesh

(Elements: 3840, Nodes: 11769)

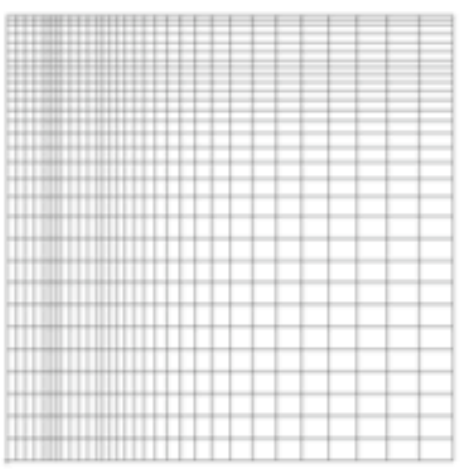

Medium mesh

(Elements: 960, Nodes: 3005)

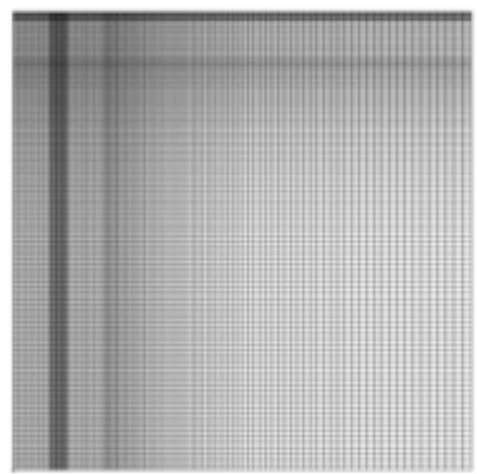

Finer mesh

(Elements: 15360, Nodes: 46577)

Figure 5. Finite element models used in the punch problem 


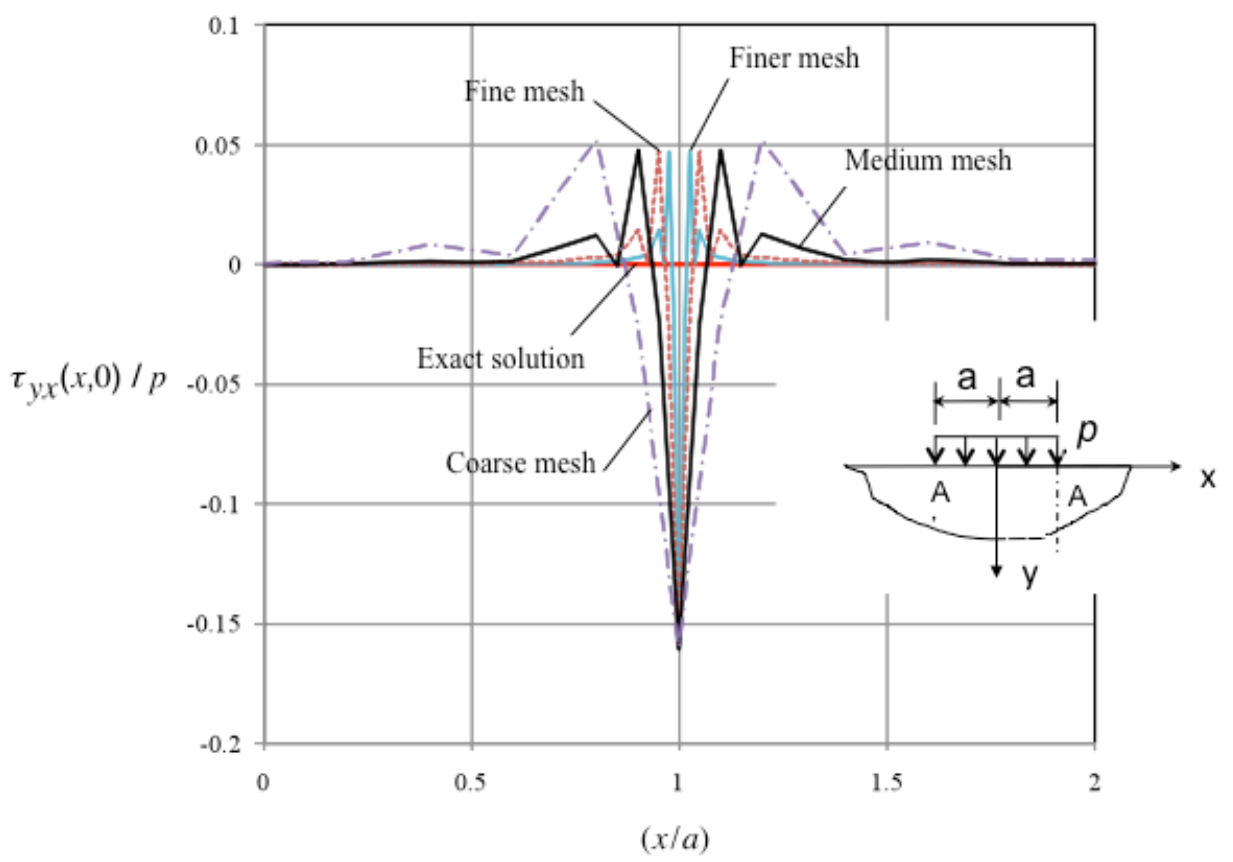

Figure 6. Shear stress $\left(\tau_{y x} / p\right)$ distribution on $y=0$ line

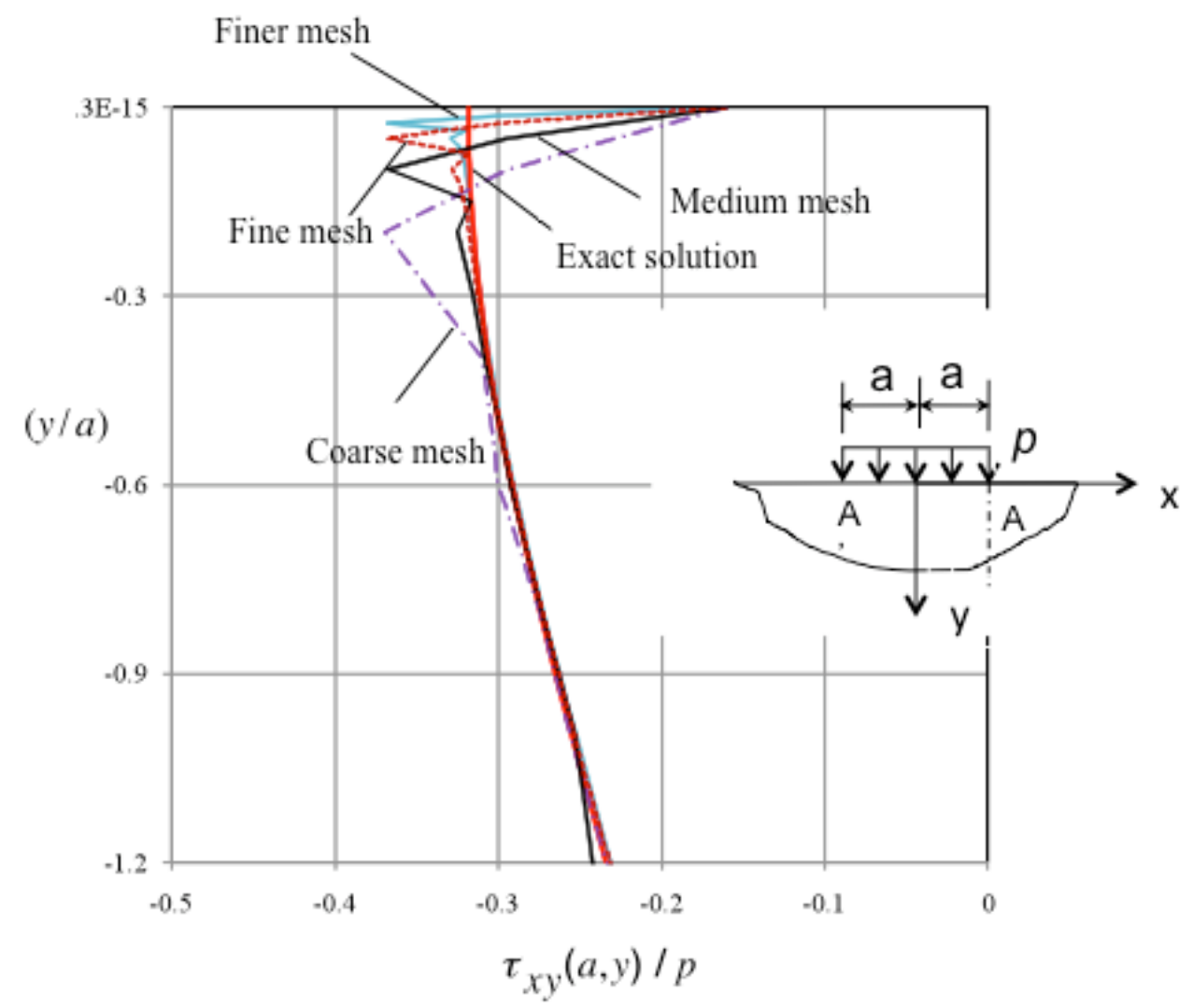

Figure 7. Shear stress $\left(\tau_{x y} / p\right)$ distribution on $x=a$ line 


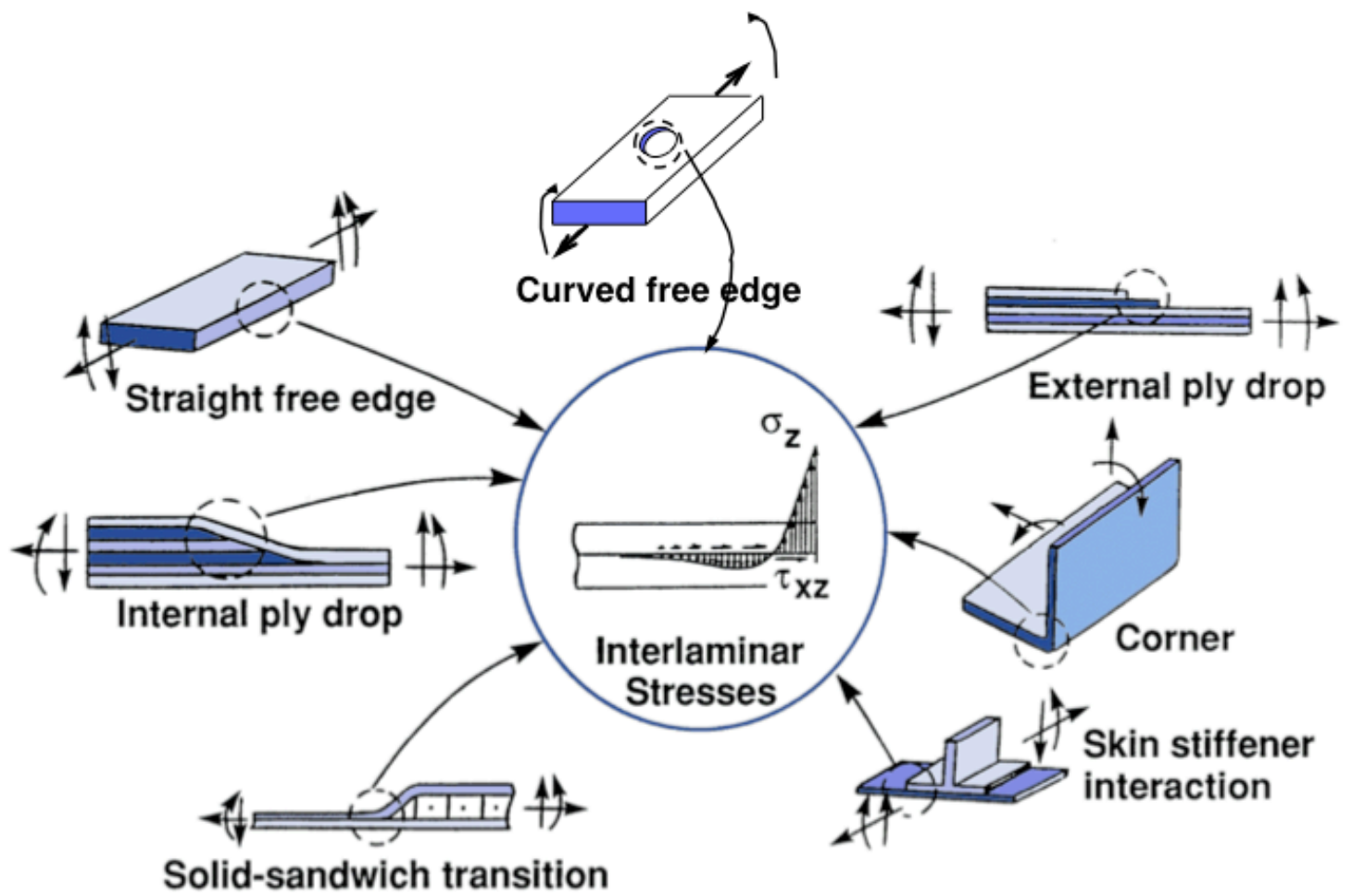

Figure 8. . Sources of delaminations at geometric and material discontinuities

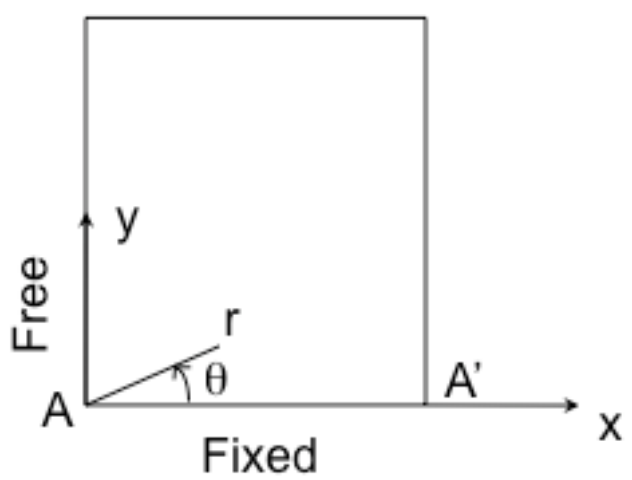

(a) Plate with fixed-free boundary conditions

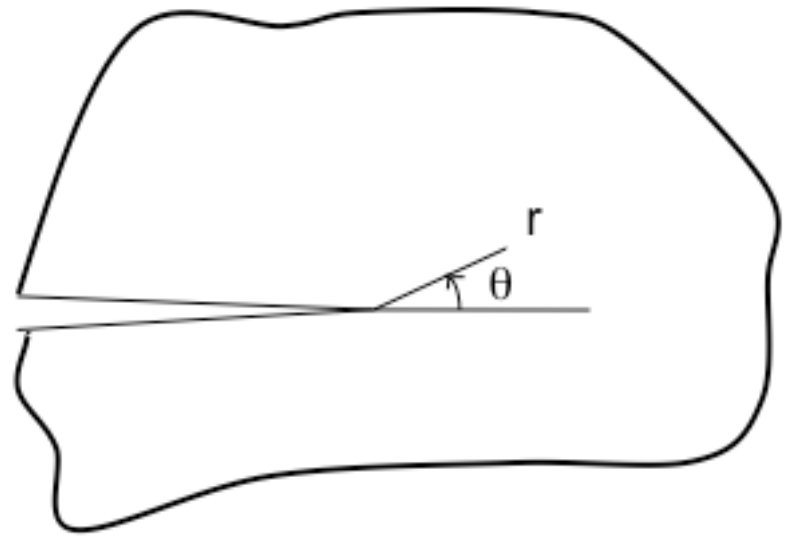

(b) Crack in a plate

Figure 9. Problems with stress singularities 

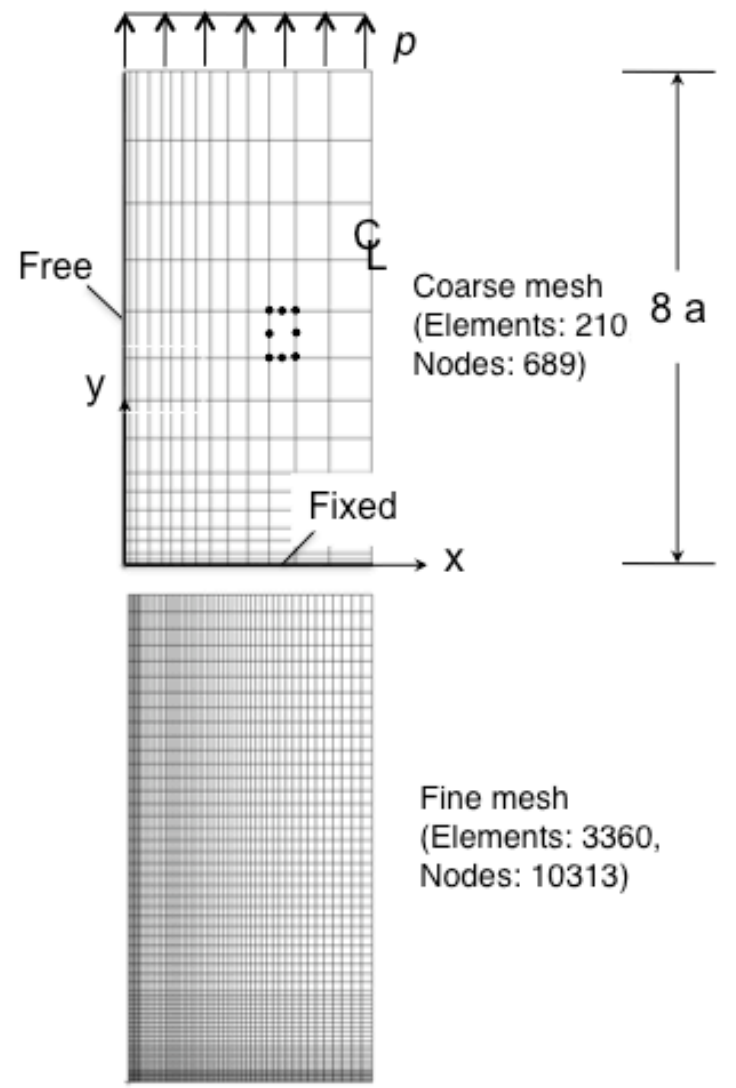

Fine mesh

(Elements: 3360,

Nodes: 10313)

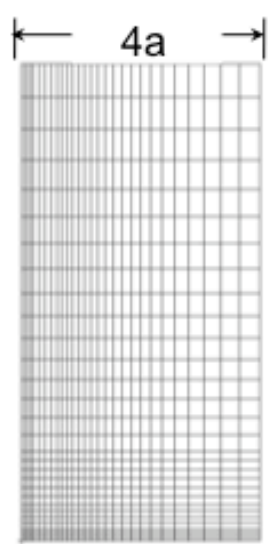

Medium mesh

(Elements: 840,

Nodes: 2637)

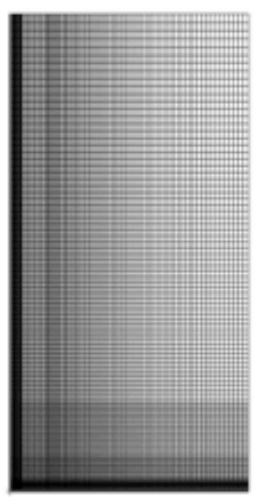

Finer mesh

(Elements: 13440, Nodes: 40785)

Figure 10. Finite element models for a fixed-free plate 


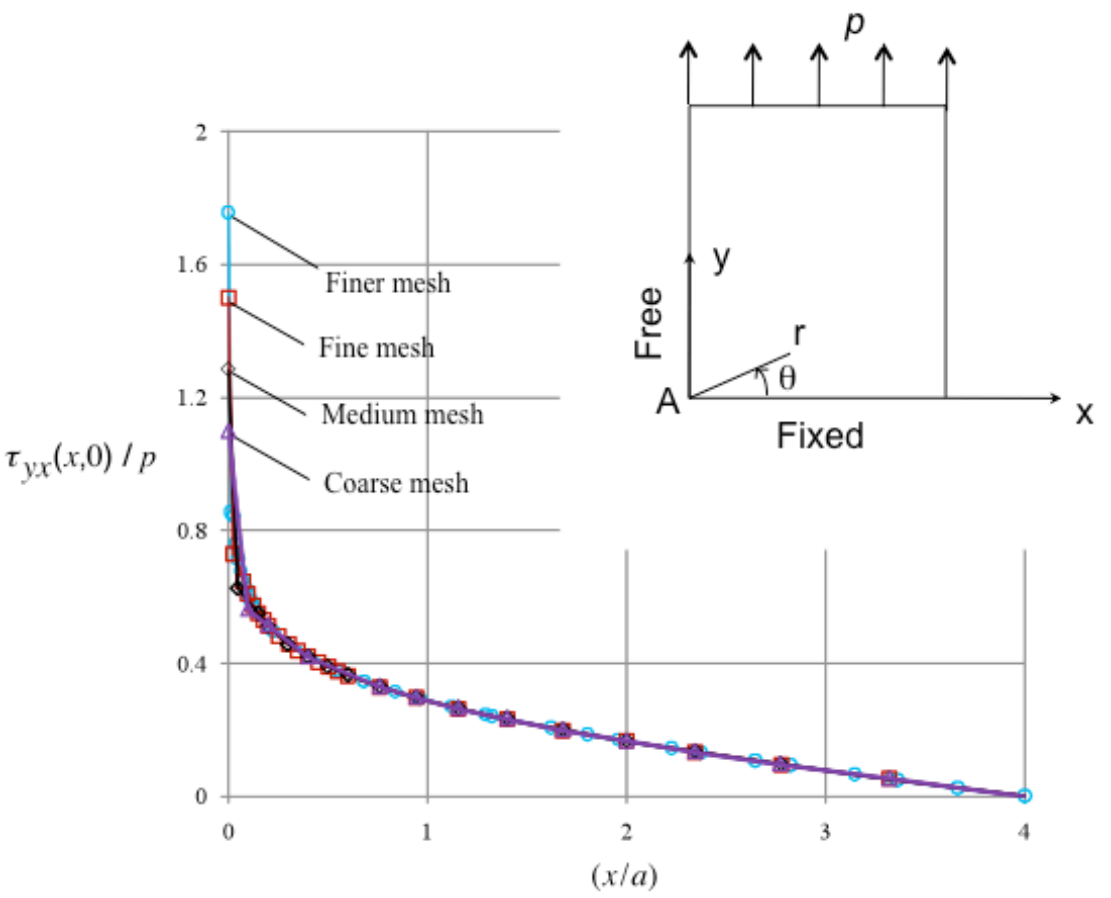

Figure 11. Shear stress $\tau_{y x}$ along the $y=0$ line

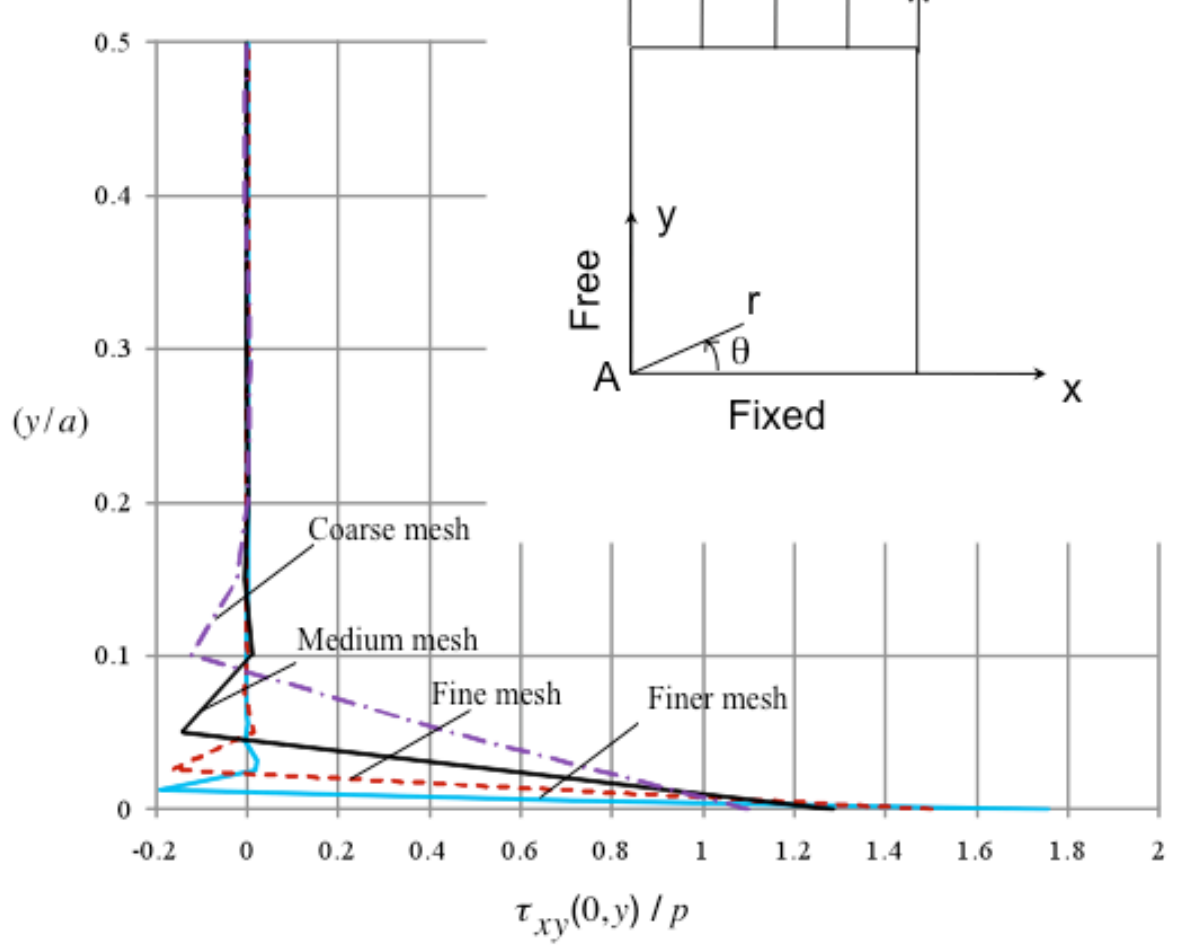

Figure 12. Shear stress $\tau_{x y}$ along the $x=0$ line

American Institute of Aeronautics and Astronautics 


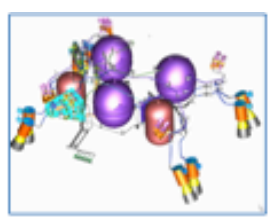

(a) Propulsion system layout

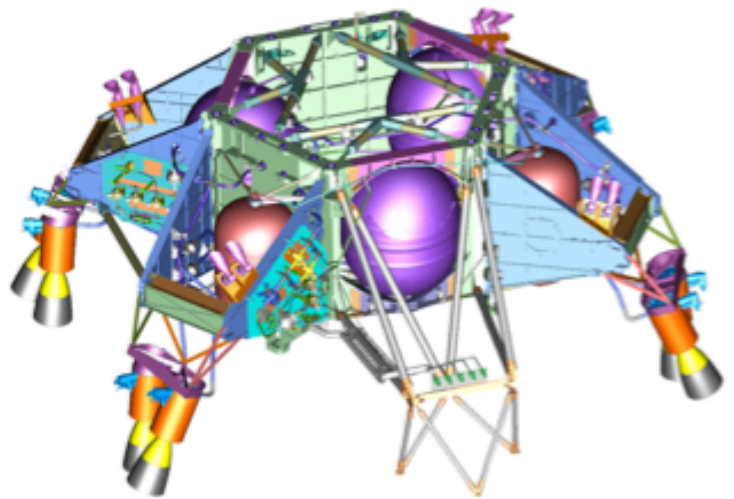

(b) Global structure

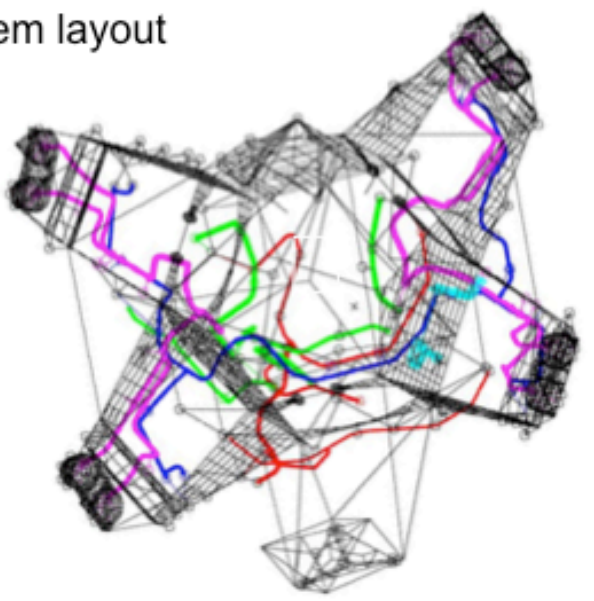

(c) Global loads finite element model

Figure 13. MSL Descent Stage

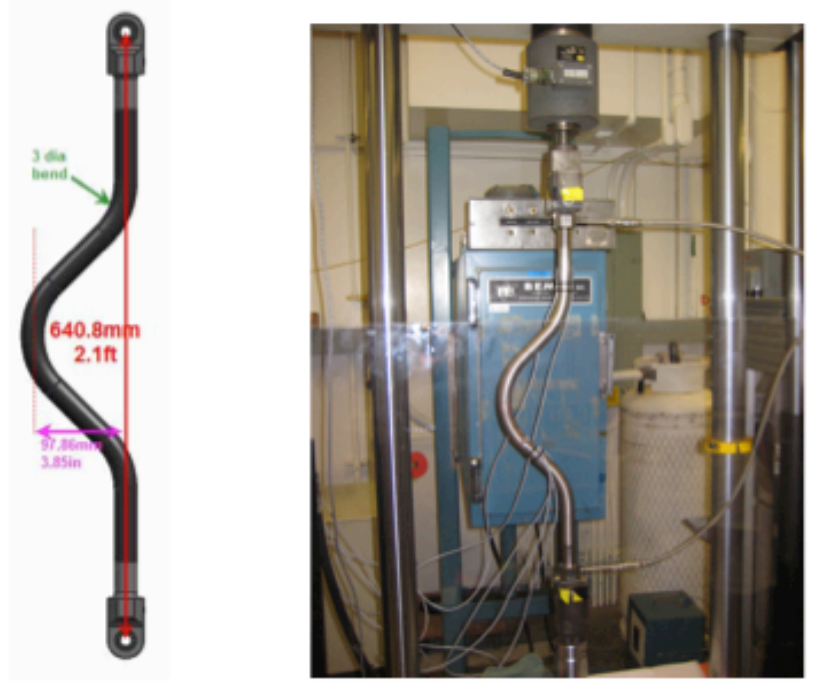

(a) Test Configuration

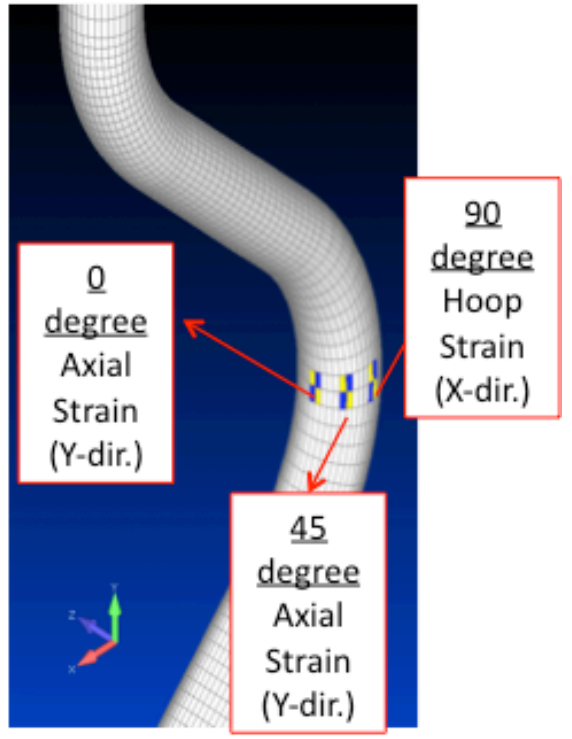

(b) Strain gage locations

Figure 14. Test Configuration of Simplified Propulsion Line 


\begin{tabular}{|l|c|}
\hline & $\begin{array}{c}\text { @ 0-150 lbs } \\
\text { Stiffness }\end{array}$ \\
\hline SS Bent Tube & lbs/in \\
\cline { 2 - 2 } Linear Analyses (Tens = Comp) & \\
\hline All shell model & 1560 \\
All beam model & 3930 \\
CBEND/beam model & $\mathbf{1 4 1 0}$ \\
& \\
Nonlinear Analyses & 1007 \\
\hline All shell model - compression & 1655 \\
All shell model - tension & \\
& \\
Test data (use best data or average) \\
\hline Compression & 1017 \\
Tension & 1426 \\
\hline
\end{tabular}

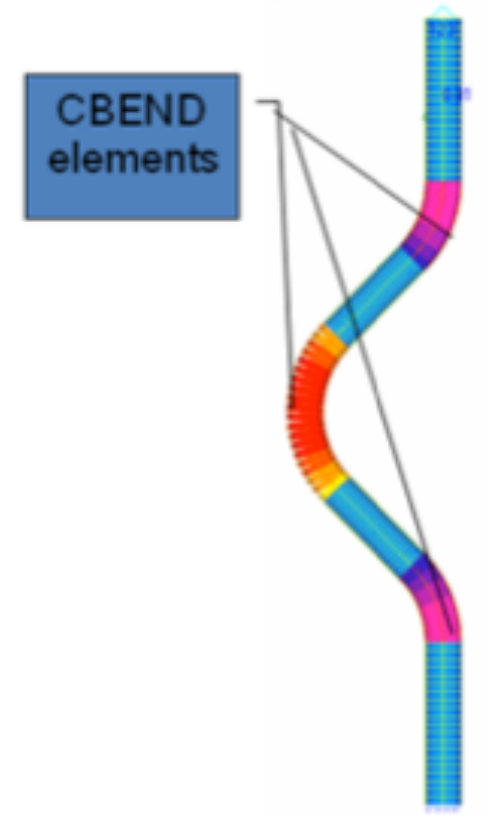

Figure 15. Stiffness Analyses and Test Results
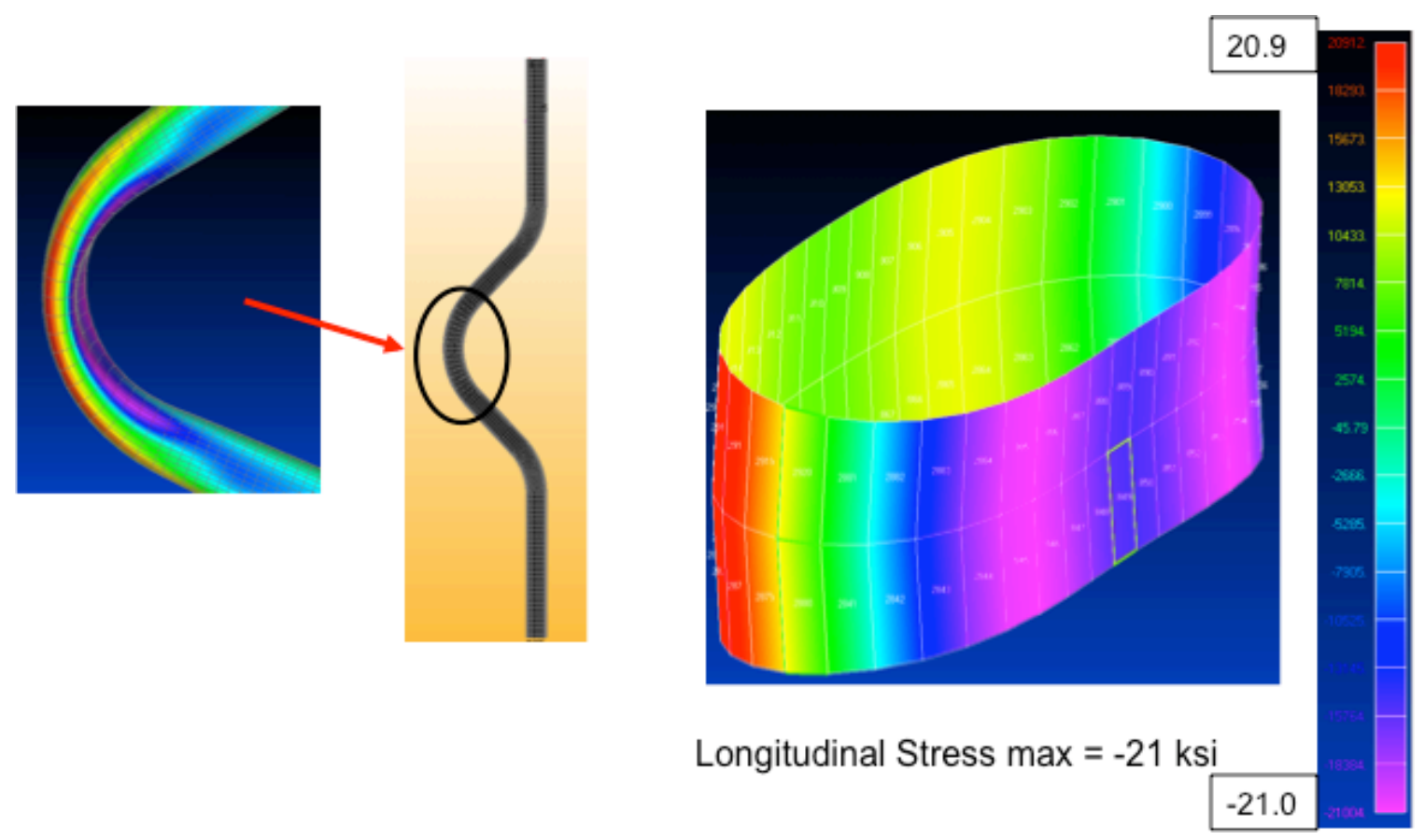

Figure16. Maximum Stress predicted by the shell model occurs at the 45 degree location 